\title{
Sars-CoV-2 Vaccines: time to consider a child-focused strategy
}

Pablo Vásquez-Hoyos ${ }^{1,2,3,4}$, Cristina De Rose ${ }^{5}$, Ivan Felipe Gutierrez ${ }^{6,7}$ Bazlin Ramly $^{8,9}$, Sebastián González-Dambrauskas ${ }^{4,10}$, Danilo Buonsenso ${ }^{5,11,12}$

${ }^{1}$ Universidad Nacional de Colobmia, Bogota, Colombia.

${ }^{2}$ Fundacion Universitaria de Ciencias de la Salud, Bogota, Colombia

${ }^{3}$ Sociedad de Cirugia Hospital de San Jose, Bogota, Colombia.

${ }^{4}$ Red Colaborativa Pediátrica de Latinoamérica (LARed Network), Montevideo, Uruguay

${ }^{5}$ Department of Woman and Child Health and Public Health, Fondazione Policlinico Universitario A. Gemelli IRCCS, Rome, Italy

${ }^{6}$ Unidad de Infectologia Pediatrica, Departamento de Pediatria, Clinica Infantil Colsbusidio, Bogota, Colombia

${ }^{7}$ Unidad de Infectologia Pediatrica, Departamento de Pediatria, Clinica Infantil Santa Maria del lago, Bogota, Colombia

${ }^{8}$ Raji Permasuri Bainun Hospital, Ipoh, Perak, Malaysia

${ }^{9}$ Paediatric Department, Children's Health Ireland Crumlin, Dubmin 12, Republic of Ireland

${ }^{10}$ Cuidados Intensivos Pediátricos Especializados (CIPe), Casa de Galicia, Montevideo, Uruguay

${ }^{11}$ Dipartimento di Scienze Biotecnologiche di Base, Cliniche Intensivologiche e Perioperatorie,

Università Cattolica del Sacro Cuore, Rome, Italy;

${ }^{12}$ Center for Global Health Research and Studies, Università Cattolica del Sacro Cuore, Roma, Italia

\section{Corresponding author:}

Danilo Buonsenso

danilobuonsenso@gmail.com 
Largo A. Gemelli 8, 00168, Rome, Italy

+390630154390

Twitter: @surf4children

Conflict of interests: nothing to declare

\begin{abstract}
With the arrival of SARS-CoV-2 vaccines, a new stage of the pandemic commenced, with new challenges ahead. During the coming months, countries will be implementing their COVID-19 vaccination programs depending on their implementation of vaccine availability and its prescription on risk stratification. Although children will not benefit from active immunization programs, now, with the beginning of the era of the anti-COVID19 vaccines, the suffering of children can no longer be ethically tolerated or neglected. The time has come to provide specific lasting strategies for children living in the COVID-19 era. Here we propose a child-focused indirect COVID-19 vaccination strategy.For better or worse, children depend on their natural caregivers (adults) and the rest of society for their well-being and achieving their full potential. We believe that including in the priority categories also those adults in close contact with children could ensure a safety net of child protection.A child-focused vaccination strategy would allow the faster return to "normality" for children and their families. Such an approach would not only enable the reopening and continuity of essential services for children but also would allow adults to return to their routine economic/productive activities.
\end{abstract}

Keywords: Covid-19, children, Sars-CoV-2 Vaccines, child-focused strategy 
With the arrival of SARS-CoV-2 vaccines, a new stage of the pandemic commenced, with new challenges ahead. During the coming months, countries will be implementing their COVID-19 vaccination programs depending on their implementation of vaccine availability and its prescription on risk stratification. In general, those considered as priority categories are "Health workers at high to very high risk of becoming infected and transmitting SARS-CoV-2 in the community transmission epidemiologic setting" and "Sociodemographic groups at significantly higher risk of severe disease or death "(1).

Since SARS-CoV-2 has had a relatively mild clinical impact on the pediatric population, children have not been considered a priority target for vaccination programs (1). Moreover, no studies have yet been launched on candidate COVID-19 vaccines for young children and therefore no safety/efficacy data are expected for this age group in the short term (1).

Although children will not benefit from active immunization programs, still a possibility exists of implementing a strategy where children (and their rights) are finally considered a priority, while guaranteeing the best impact on the whole community.

Physical distancing measures designed to decrease or prevent community transmission of SARSCoV-2 have significantly affected children's and families' quality of life. The prolonged interruption of child education services has led not only to educational poverty (2) but also deprived children of the learning and social experiences necessary for their full development: cognitive and psychic development, improvement of critical thinking, and social skills $(2,3)$. Schools and out-of-school settings (after schools, support centers for children with special needs, services for socio-cultural and recreational activities, health services) also provide several additional important benefits for the health and well-being of children. Social interactions, provision of health services delivery such as vaccinations, meals, and shelter from unstable or unsafe home environments are some examples. The extent of learning loss, and the additional functions of school and out-of-school environments and their impact on life prospects are far greater for children living in poverty or otherwise disadvantaged groups $(4,5)$. The closure of child activities had an obvious impact on parents' lives and their working routine, particularly on women that mostly care about their children (6). In resource-limited settings, such as in many regions in Latin America school interruption has been more pronounced, and even virtual learning has not been a possibility.

Now, with the beginning of the era of the anti-COVID19 vaccines, the suffering of children can no longer be ethically tolerated or neglected. The time has come to provide specific lasting strategies for 
children living in the COVID-19 era. Here we propose a child-focused indirect COVID-19 vaccination strategy.

For better or worse, children depend on their natural caregivers (adults) and the rest of society for their well-being and achieving their full potential. We believe that including in the priority categories also those adults in close contact with children (such as those who belong to the school and nonschool child-educational services, grandparents which may support families in caring for young children) could ensure a safety net of child protection. In particular, teachers, support teachers, and adults working on centers for children with special needs or recreational services should have priority in the programs of vaccination. This will facilitate the safer full reopening of school and non-school educational settings and will provide community and governmental confidence in reopening school settings. Local decisions regarding vaccines prioritization could be based according to regional necessities, the possibility of distance learning settings, and social disparities, among others.

A child-focused vaccination strategy would allow the faster return to "normality" for children and their families. Such an approach would not only enable the reopening and continuity of essential services for children but also would allow adults to return to their routine economic/productive activities.

SARS-CoV-2 vaccine delivery is certainly a complex strategy and open to discussions, since we do not have studies suggesting the most effective approach. In any case, discussing strategies aimed at the protection of early childhood from the indirect consequences of the pandemic is definitely a priority. The history of modern vaccination started in 1796 when Edward Jenner performed the first vaccination of James Phipps, an 8-year-old boy (7). Our history demonstrates that humanity has a historic debt to children regarding vaccination development and expansion saving millions of lives throughout 2 centuries. If children resulted to be relatively spared from COVID-19 severe disease and then do not deserve the first place to receive the COVID19 vaccines, then the pandemic is an excellent opportunity to put children at the center under indirect protection of a safety social net around them. Children should always be the core of our societies since they are naturally one of the most vulnerable populations. We can now translate this concept into actions.

\section{Acknowledgments}

We are grateful to dr. Lucia Alonso for her intellectual support and suggestions 


\section{References}

1. https://www.who.int/docs/default-source/immunization/sage/covid/sage-prioritizationroadmap-covid19-vaccines.pdf?Status=Temp\&sfvrsn=bf227443_2

2. Christakis DA, Van Cleve W, Zimmerman FJ. Estimation of US Children's Educational Attainment and Years of Life Lost Associated With Primary School Closures During the Coronavirus Disease 2019 Pandemic. JAMA Netw Open. 2020 Nov 2;3(11):e2028786. doi:10.1001/jamanetworkopen.2020.28786. PMID: 33180132; PMCID: PMC7662136.

3. Orben A, Tomova L, Blakemore SJ. The effects of social deprivation on adolescent development and mental health. Lancet Child Adolesc Health. 08 2020;4(8):634-640. doi:10.1016/S2352-4642(20)30186-3

4. Protecting the most vulnerable children from the impact of coronavirus: an agenda for action. New York (NY): United Nations Children's Fund; 2020 (https://www.unicef.org/coronavirus/agenda-foraction?fbclid=IwAR1YLd4B5gXm9506u0gMlCwK-gMAD9NgDAVDwUM2Vdrrqpo2j3z8B-hYFo, accessed 15 October 2020).

5. Buonsenso, D.; Roland, D.; De Rose, C.; Vásquez-Hoyos, P.; Ramly, B.; Nandipa ChakakalaChaziya, J.; Munro, A.; González-Dambrauskas, S. Schools Closures during the COVID-19 Pandemic: A Catastrophic Global Situation. Preprints 2020, 2020120199 (doi: 10.20944/preprints202012.0199.v1).

6. Palmer J; Highlights 2020 winning contributors. Highlights 2020: framing health stories. Lancet. 2021 Dec 19;396(10267):1957-1971.

7. Riedel S. Edward Jenner and the history of smallpox and vaccination. Proc (Bayl Univ Med Cent). 2005 Jan;18(1):21-5. 